\title{
Biocompatible Antidermatophytic Scaffolds (TfG-Nf) for Controlled and Impressive Management of Topical Tinea Diseases
}

\section{Shashi Kiran Misra ${ }^{1 *}$, Himanshu Pandey², Kamla Pathak ${ }^{3}$ and Sandip Patil $^{4}$}

${ }^{1}$ University Institute of Pharmacy, CSJMU, Kanpur, India

${ }^{2}$ Central University of Higher Tibetan Studies, Varanasi, India

${ }^{3}$ Faculty of Pharmacy, Uttar Pradesh University of Medical Sciences, Saifai, Uttar

Pradesh, India

${ }^{4}$ E-Spin NanoTech Private Ltd., SIDBI Innovation and Incubation Center, Indian

Institute of Technology, Kanpur, India

*Corresponding Author: Shashi Kiran Misra, University Institute of Pharmacy, CSJMU, Kanpur, India.
Received: May 24, 2021

Published: June 03, 2021

(C) All rights are reserved by Shashi Kiran

Misra., et al.

\begin{abstract}
Purpose: The rationale behind this study was to develop graphene based tolnaftate conjugate (Tf G) and embed it within scaffolds composed of biocompatible Eudragit polymers for controlled and impressive antitinea activity.

Methods: Developed Tf G conjugate was entrenched in 20\% w/v polymeric solution (ERL100/ERS100) and nonwoven scaffolds through E-spin (electrospinning) technology were fabricated. Assorted analytical techniques i.e. FESEM, FTIR, XRD and DSC were employed to characterize and assessment of scaffolds. Sessile drop and Dialysis bag methods were utilized for the determination of their hydrophilicity and drug release behavior. In vitro fungal study was performed on enormously virulent strains of tinea infections i.e. zoophilic Microsporum fulvum and anthropophilic Trichophyton rubrum. Animal study was performed on Trichophyton rubrum diseased Swiss albino mice for seven days.

Results: High payload of Tf on G was perceived by the virtue of extensive surface area of G and process of physisorption. Surface morphology analyzed that TfG-Nf were nano-dimensional (90-120 nm), uniform, defect-free and possessing numerous pores. Tremendous swelling capacity and pronounced hydrophilicity were obtained that gratified crucial benchmarks for scaffolds to alleviate tinea infections. In vitro drug release data interpreted controlled diffusion of drug for 8 hrs. Microdilution assay exhibited remarked eradication (99.356\%) of viable cells of T. rubrum after $96 \mathrm{hrs}$. Animal study on infected Swiss albino mice exposed better antitinea activity of TfG-Nf amid new growth of hair at infected site.

Conclusion: Fabricated TfG-Nf exhibited superior in vitro and in vivo action than Tf- Nf scaffolds and offered promising topical drug delivery approach to mitigate superficial bugs and heal up at infected regions. Findings also endorse newer biomedical application of graphene in designing wound dressings (bandages/scaffolds) materials.
\end{abstract}

Keywords: Tolnaftate; Graphene; Nano-dimensional; Scaffolds; Tinea Disease; Eudragit; Trichophyton rubrum 


\section{Abbreviations}

Tf: Tolnaftate; G: Graphene Nanoplatelets; Tf G: Tolnaftate Graphene Conjugate; Tf Nf : Tolnaftate Scaffolds; TfG-Nf: Conjugate Loaded Scaffolds; G Nf: Graphene Nanoplatelets Loaded Scaffolds; ERL: Eudragit RL100; ERS: Eudragit RS100; PEG 400: Polyethylene Glycol 400; WCA: Water Contact Angle; MTCC: Microbial Type Culture Collection; DMSO: Dimethyl Sulfoxide; SDA: Sobouraud Dextrose Agar; NCCLS: National Committee for Clinical Laboratory Standards; XRD: X-ray Diffraction; FTIR: Fourier- transform Infrared Spectroscopy; DSC: Differential Scanning Calorimetry; FESEM: Field Emission Scanning Electron Microscopy; ECM: Extracellular Matrix; CDR: Cumulative Drug Release.

\section{Introduction}

Till the $20^{\text {th }}$-century mere bacterial epidemic was considered the threatened cause of mortality but late 1960 expressed the drastic rise of menace dermatophytes provoking Tinea infections or skin bugs. Despite enormous dermal remedies, their poor efficacy and nonlocalized impact at the site of infection challenge the researchers in clinical practices. Tinea infections have been positioned as $4^{\text {th }}$ of maximum common transmissible skin ailment in the last ten years, exaggerated $1 / 4^{\text {th }}$ biosphere's inhabitants [1] and proliferate at the site of host with the weakened immune system [2]. Tineas causative pathogens are keratinophilic fungi [3], comprise species of Microsporum, Trichophyton, Epidermophyton and Achiron that interrupt skin keratin through discharging proteolytic enzymes [4]. General remedies offer efficacy at a prelim stage but failed at a progressive stage due to their compromised pharmacokinetic parameters [5]. Patients having poor immunity, diabetes, cystic fibrosis and malignancy are more susceptible to Tinea infections. The patients receiving prolonged massive doses of antibiotics, corticosteroids, and cytotoxic agents or dependent upon parenteral nutrition, become more prone/susceptible to tinea infections [6]. Although, application of the broad spectrum antibiotics destruct the prokaryotic bacterial cells effectively and lucratively cure the bacterial skin diseases, leaving no harmful effect over the host tissue while the eukaryotic cell set up of the fungal pathogen and its host, makes treatment relatively difficult [7]. The prescribed antibiotics also leave a toxic effect over the host tissue as their weak doses create drug resistance in the pathogen, and strong dose leaves harmful after effects on the host, thus prove to be either too weak or too toxic remedy. Hence the escalating number of Tinea infections can only be brought under control by designing a precise bioactive dosage that would be curative over prophylaxis.

A perusal of relevant literature reveals that the use of nano-dimensional scaffolds tends to create greater interconnectivity, the feature of inherent small pore dimension results in the high surface area to ensure a controlled release of drug at the site of infection [8]. By mimicking the native extracellular matrix, electrospunned scaffolds modulate both physically and chemically, the cell adherence and differentiation. It retains biocompatible properties and efficiently promotes tissue regeneration [9]. The ability to embed several bioactive agents into their network increases their potential in wound dressings and tissue engineering [10]. Moreover, this approach unveils auxiliary features to nurture localized effect of the drug that combines with the pathogen only. Thus, such dosage form strictly trails over localized area and restricts to escalation of drug into surrounding healthy tissues of the host.

Thiocarbamate derivative tolnaftate (Tf) our model drug, frequently used to manage tinea diseases [11]. Biopharmaceutical classified type IV tolnaftate is a strong hydrophobic drug that leads deprived permeability and hence tender confront for topical dosage formulations [12]. Globally, tolnaftate is marketed in form of gel, cream, and aerosol spray, but most of them show signs of stickiness, irritation, and reduced penetration thus necessitate prolong therapy for complete alleviation of Tinea infections with scanty patient compliance.

The research on graphene for wound dressing and cell culture is a relatively new approach that deserves special attention. Enormous studies in this ground so far demonstrated that graphene is antibacterial [13], accelerate the growth differentiation [14] and proliferation of mammalian cells $[15,16]$ and hence holds immense perspective in tissue engineering, regenerative medicine, and new biomedical fields. Here, Graphene is selected as an effective nanocarrier owing to its potential for crossing the plasma membrane and promoting the cellular uptake of the pathogen at the infected site. Nobel Prize conquerors Andre Geim and Konstantin Novoselov had done meticulous work on charismatic graphene in 2010, avowed that one millimeter of graphite composed of three million layers of graphene stacked to one another, weakly detained together and therefore moderately easy to tear off and split [17]. Graphene has unique hierarchical and physicochemical properties 
including a high surface area $\left(2630 \mathrm{~m}^{2} / \mathrm{g}\right)$, extraordinary electrical and thermal conductivity (mobility: $20,000 \mathrm{~cm}^{2} \mathrm{~V}^{-1} \mathrm{~s}^{-1}$ ) owing to the several pie electron that facilitates appropriate sites for development of conjugates possessing sufficient mechanical strength [18]. Both sides of each graphene sheet are accessible for drug binding [19] and they possess extraordinary electrical, optical, thermal, mechanical properties and offer interesting cytotoxic and antimicrobial action [20]. The unique 2D shape and single sheet of graphene nanoplatelets $(G)$ are considered as gates or lattices for holding therapeutics and control their release in sequential manner. The high surface area and both planes of graphene are available for a high payload of water insoluble drugs [21].

We herein report controlled drug delivery and improved efficiency of Eudragit scaffolds loaded with antifungal tolnaftate - graphene conjugate for the absolute eradication of Tinea infections. A blend of Eudragit (polymethacrylate) polymers are been selected for production of scaffolds owing to their proficient film forming capacity, biocompatibility and anti-tacking property on the skin [22]. Customized and controlled drug release [23,24] from scaffolds would be achieved due to the erratic water permeability of both polymers. Polyethylene glycol (PEG 400) is added to the polymeric solution to shape out nano -ranged framework [25], to render flexibility sufficient mechanical strength at the site of infection. Furthermore, PEG 400 ameliorates better dispersibility of nanoconjugate in a polymeric solution [26], enhances solubility of loaded nanoplatelets and thus must facilitate bioactive efficiency $[27,28]$. It also hampers opsonization or immunogenicity of graphene based nanoplatelets via shielding against the immune system [29].

\section{Experimental}

\section{Materials}

Tolnaftate was received from Belco Pharmaceuticals, Haryana. Graphene nanoplatelet was purchased from Reinste Pvt. Ltd, Delhi. Tinea causatives T. rubrum (MTCC 7859) and M. fulvum (MTCC 2839) were acquired from MTCC, Chandigarh, India. Eudragit RL100 and Eudragit RS 100 (150,000 Da) were obtained as gift samples from Evonik (Germany). RPMI 1640 and MOPS were procured from Sigma-Aldrich Chemicals Pvt. Ltd, New Delhi. SDA was bought from HiMedia Laboratories Pvt. Ltd, Mumbai.

\section{Development of Tf G and fabrication of TfG-Nf scaffolds}

Herein, $1 \mathrm{mg} / \mathrm{ml}$ Tf was encumbered efficiently on the acetone dispersion of $\mathrm{G}$ via the wet chemical route to acquire uniform $\mathrm{Tf}$ G conjugate following a few seconds' sonications and 15,000 rpm ultracentrifugations. Developed Tf G conjugate was collected and loading efficiency was estimated through UV-Visible spectrophotometer applying straight-line equation. Furthermore, $20 \% \mathrm{w} / \mathrm{v}$ polymeric solution (ERL100: ERS100,1:1) was prepared in methanol and dimethylacetamide solvent mixture $(3: 2)$. To this, independently Tf, G, and Tf $\mathrm{G}$ (each $1 \% \mathrm{w} / \mathrm{v}$ ) were added followed by $1 \%$ w/v PEG 400 to ameliorate physicochemical properties as stable fabrication of scaffolds by virtue of surface modifier and preventing the inter-particular aggregation of the dispersed conjugate in polymeric solution. Prepared each dispersion was loaded separately into a $10 \mathrm{ml}$ plastic syringe armed with a piercing needle. The syringe was positioned in a syringe pump and was coupled to a voltage supply of $15 \mathrm{kV}$ (Figure 1 ). The repulsive electrical force pulls the pendant drop developed at needle making a Taylor Cone when the voltage was increased [30] and reaches to the bras collector in form of fine scaffolds within nanoseconds in E-Spin, electrospinning machine.

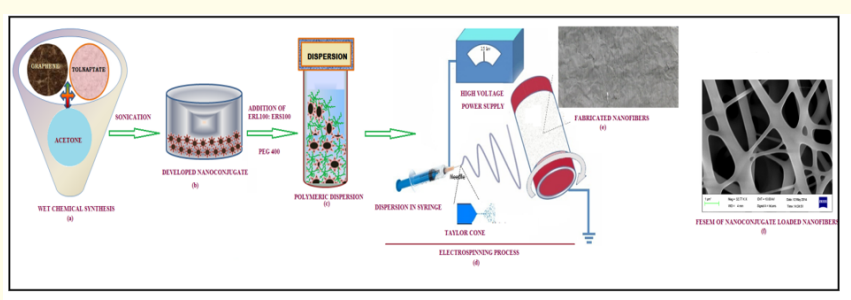

Figure 1: Schematic illustration of the development of conjugate and fabrication of nonwoven scaffolds.

\section{Characterization}

Fourier transform infrared spectrometer (FTIR- ATR, Bruker Optik, Germany) containing a DLaTGS detector along with a germanium internal reflection element was employed at range 3500$500 \mathrm{~cm}^{-1}$ to recognize functional groups existing in individual compounds and compare spectrum of Tf, G, Tf G and fabricated TfG Nf scaffolds. X-Ray Diffraction studies ( $X^{\prime}$ Pert PRO, PAN analytical, Netherland) were used to determine the $2 \theta$ range for the above cited compounds at $40 \mathrm{mV}$ and $300 \mathrm{~mA}$. Differential scanning calo- 
rimetry (DSC) was employed to determine thermal effect on compounds at a temperature range from $30^{\circ} \mathrm{C}$ to $600^{\circ} \mathrm{C}$ at a required heating rate $\left(10^{\circ} \mathrm{C} / \mathrm{min}\right)$.

Scanning electron microscopy (FESEM quanta 200, Zeiss, Germany) was employed to characterize the morphology of blank polymeric (Nf), Tf loaded (Tf-Nf), G loaded (G Nf), and Tf G loaded (TfG-Nf) scaffolds. Initially, all the samples were one minute gold sputter-coated $(20 \mathrm{~nm})$ to render them electrically conductive. Gold treated scaffolds were kept in the microscope compartment to which a high vacuum was provided and their pictures were captured at $15 \mathrm{kV}$ excitation voltage. The FESEM images were analyzed by using the software Image J and their surface architecture (texture, shape, and diameter) was demonstrated [31]. The average mean diameter and standard deviations of the fabricated scaffolds were measured from 50 random dimensions at a magnification of 1000x.

Static water contact angle (WCA) of fabricated scaffolds was measured through sessile drop method to define extent of hydrophilicity using Rame-Hart contact angle goniometer [32]. Approximately $25 \mu \mathrm{L}$ of deionized water was allowed to fall over the desiccated part of scaffolds with an assembled microsyringe. WCA was observed after 60 seconds of incubation and average contact angles were reported of five successive values.

The swelling index plays significant part during drug release from the architecture of scaffolds. $2 \mathrm{~cm}^{2}$ of each fabricated scaffolds were kept in petri dish comprising phosphate buffer $\mathrm{pH} 6.8$ media $(10 \mathrm{ml})$ and incubated for $24 \mathrm{hr}$ at $37^{\circ} \mathrm{C}$. Adhered excess media on their surface of scaffolds was removed carefully with Whatman filter paper. Swelled scaffolds were weighed till persistent weight was achieved and the average swelling index of scaffolds was estimated.

\section{In vitro drug release}

The dialysis bag method was preferred for the determination of in vitro drug release utilizing 2.4 nm HIMEDIA ${ }^{\circledR}$ LA 387 Dialysis Membrane-50 [33]. Literature envisaged that alteration of skin $\mathrm{pH}$ from acidic to alkaline occurs after tinea infection [34], henceforth drug release from Tf-Nf and TfG-Nf (each of $5 \mathrm{~cm}$ ) was considered out in different release medium pH 3.8, 6.8 and 9.0. Test aliquots $(5 \mathrm{ml})$ were withdrawn and estimated for amount of drug release through UV-spectrophotometer ( $\lambda \max 258 \mathrm{~nm})$.
Preparation of fungal strain inoculum

The seven days old fungal strains of T. rubrum (MTCC 7859) and M. fulvum (MTCC 2839) were subcultured on SDA at room temperature. The stock inoculum suspensions of both strains were made in DMSO solvent and the optical density of prepared suspension was attuned through SPECTRAMAX plus 384 at wavelength of 530 $\mathrm{nm}$. The composition and turbidity of each inoculum were compared with 0.5 McFarland to produce strength of $1-5 \times 10^{5}$ cells $/ \mathrm{ml}$.

\section{Localized effect}

An equal dimension of the nanofibrous scaffold was sterilized under laminar airflow through UV light and placed carefully with the help of sterile forceps on surface of the one week old subculture of fungal strains (T. rubrum (MTCC 7859) and M. fulvum (MTCC 2839) inoculated SDA plates, followed by incubation for 24 hours at $30^{\circ} \mathrm{C}$. After incubation, each plate was investigated for localized effect and leaching behavior of bioactive compound from scaffolds in surrounding media [35].

\section{In vitro antifungal assay}

The Microdilution method was carried out to evaluate inhibitory concentrations of fabricated scaffolds towards tinea infection ensuing CLSI guidelines [36]. Selected dermatophytes were treated with RPMI1640, MOPS (0.165 mol/L) and L-glutamine (2\%) to yield $1 \times 10^{5} \mathrm{CFU} / \mathrm{mL}$ in a pre-sterilized 96 well microtiter plate [37]. Thereafter, dispersions of fabricated scaffolds (0.01-1.25 $\mathrm{mg} / \mathrm{ml}$ ) in DMSO were added into virulent strains treated 96 well microtiter plate [38,39]. Minimum inhibitory concentrations for dermatophytes were analyzed at $530 \mathrm{~nm}$ through Spectramax Plus 384 spectrophotometer (Molecular device, USA).

\section{In vivo study}

Swiss Albino mice (male, 4-5 weeks old with average weight 20$25 \mathrm{~g}$ ) were selected after getting ethical approval by animal ethical committee, India (BU/Pharm/IAEC/15/01). Topical application of fabricated scaffolds was commenced on each group of Swiss albino mice categorized as Group I (untreated/control), Group II (treated with marketed preparation), Group III (treated with Nf), Group IV (treated with Tf Nf), Group V (treated with G Nf) and Group VI (treated with TfG-Nf).

Initially, skin hairs of each mouse were removed out through applying common hair removing cream and conidial suspension of 
T. rubrum inoculum was applied on their hair shaven skin to cause tinea corporis, a kind of dermatophytosis. The treatment was started after the appearance of the infection and continued until complete recovery from the infection was achieved [40]. After infection, the extent of mycelia growth of the inoculated (T. rubrum) spores on the dorsal site of mice was inveigled in the form of scores ranged from 0-4 [41].

\section{Results and Discussion}

\section{Fabrication of TfG-Nf scaffold}

Graphene (G) based Tf G nanoconjugate was successfully developed via the wet chemical route. Enormous surface area of Graphene (G) offered ample basal plane via П- П stacking thus facilitated plentiful physical adsorption of Tf. Through electrospinning technique (E- Spin, Nanotech), equal blend of 20\% w/v ERL100: ERS 100 polymeric solution comprising blank (Nf), Tf loaded (TfNf), G loaded (G-Nf) and Tf G loaded (TfG-Nf) scaffolds were fabricated under controlled parameters of flow rate $(5 \mu \mathrm{l} / \mathrm{min})$, applied voltage $(15 \mathrm{kv})$ and optimum distance $(12 \mathrm{~cm})$ between syringe tip and collector.

\section{Characterization}

Comparative vibrational analysis of all ingredients and scaffolds is portrayed in figure 2a. Chemical structure of Tf demonstrated phenyl and naphthalene ring as presence of $\mathrm{C}=\mathrm{C}$ stretching mode at $1420 \mathrm{~cm}^{-1}$, which is evident for interaction of charge transfer amid donor and receiver groups, responsible for fungicide activity. The $\mathrm{C}-\mathrm{H}$ stretching (asymmetric) of methyl group was perceived at $3095 \mathrm{~cm}^{-1}$ whereas the symmetric $\mathrm{C}-\mathrm{H}$ stretching was assessed at $2875 \mathrm{~cm}^{-1}$ (Figure 2a). A precise peak at $1500 \mathrm{~cm}^{-1}$ was observed due to $\mathrm{C}-\mathrm{N}$ stretching vibration. The presence of meta disubstituted phenyl ring in the structure of Tf was perceived at $1260 \mathrm{~cm}^{-1}$ as weak bands while at $1205 \mathrm{~cm}^{-1}$ stretching mode of $\mathrm{C}=\mathrm{S}$ was projected. FTIR spectra of G displayed presence $\mathrm{C}-\mathrm{H}$ stretching of methyl group at $2950 \mathrm{~cm}^{-1}$. Strong $\mathrm{O}-\mathrm{H}$ vibrations from oxygen functionalities were exhibited at $3450 \mathrm{~cm}^{-1}$, while a weak $\mathrm{C}=0$ stretching vibrations was also located at $1650 \mathrm{~cm}^{-1}$. C-OH stretching vibrations in the structure of $\mathrm{G}$ were present at $1250 \mathrm{~cm}^{-1}$ these all suggested possibility of occurrence of carboxyl groups there. IR spectra of Tf G conjugate exhibited asymmetric $\mathrm{C}-\mathrm{H}$ stretching at $3250 \mathrm{~cm}^{-1}$ along with one distinct peak at $1260 \mathrm{~cm}^{-1}$ due to functional group $\mathrm{C}=\mathrm{S}$ group of $\mathrm{Tf}$. Moreover, another $\mathrm{O}-\mathrm{H}$ stretching and $\mathrm{C}=\mathrm{O}$ stretch- ing peaks present in G were seemed at $3200 \mathrm{~cm}^{-1}$ and $1690 \mathrm{~cm}^{-1}$ respectively, thus signifying the development of $\mathrm{Tf} G$ conjugate.

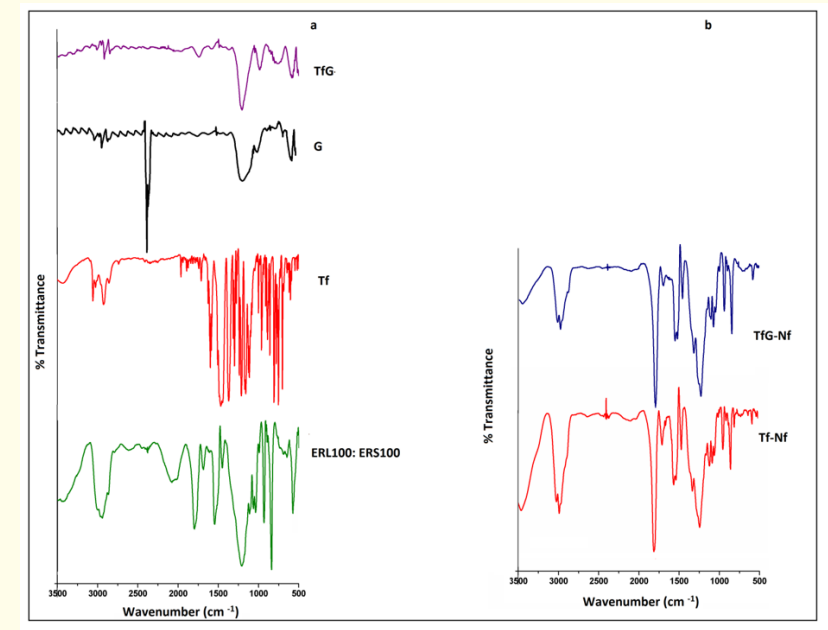

Figure 2: Compiled IR spectra of Eudragit polymers (ERL100/ ERS100), drug Tf, graphene nanoplatelets G, developed Tf-G nanoconjugate (a), scaffolds Tf-Nf and TfG- Nf (b).

IR spectra of TfG-Nf revealed that maximum peaks of Tf were either shifted or appeared to decrease in intensity, might be due to either polymeric interaction between $\mathrm{C}=0$ groups of ERL 100/ ERS 100 and the C-O functional group of Tf, or the hydrogen bonding between unbound hydrogen present in quaternary ammonium groups of polymethacrylate polymers and carbonyl groups present on $\mathrm{G}$ (Figure $2 \mathrm{~b}$ ). A common peak at $1250 \mathrm{~cm}^{-1}$ owing to functional group $\mathrm{C}-\mathrm{OH}$ (stretching) appeared in the IR spectra of framework TfG-Nf, graphene G and nanoconjugate Tf G confirming their similar integrity. Presence of quaternary ammonium group of Eudragit polymers was revealed by a distinct peak at $3050 \mathrm{~cm}^{-1}$ from FTIR of scaffolds TfG-Nf, and Eudragit polymers. Furthermore, a strong peak at $1280 \mathrm{~cm}^{-1}$ and $1090 \mathrm{~cm}^{-1}$ were obtained in the spectra of TfG-Nf by the occurrence of $\mathrm{O}-\mathrm{H}$ and $\mathrm{C}-\mathrm{O}-\mathrm{H}$ functional groups in polyethylene glycol (PEG 400). Additionally, the bending peak of $\mathrm{C}-\mathrm{H}$ and $\mathrm{C}-\mathrm{H}$ stretching was apparent at $1460 \mathrm{~cm}^{-1}$ and $3441 \mathrm{~cm}^{-1}$ respectively, suggesting the presence of PEG 400 in fabricated scaffolds. 
The powder XRD spectra revealed that Tf showed different peaks at $6^{\circ}, 9^{\circ}, 11^{\circ}, 17^{\circ}, 23^{\circ}$ and $26^{\circ}$ explaining its crystalline state. A sharp peak at $26^{\circ}$ obtained of $\mathrm{G}$ which became a boarder in XRD of nanoconjugate $\mathrm{Tf} \mathrm{G}$, maybe due to molecular dispersion or noncovalent interaction between Tf and G (Figure 3a). Distinct peaks were absent from XRD spectra of TfG-Nf scaffolds in figure $3 \mathrm{~b}$ which may be attributed to the interaction that occurred between metastable crystalline polymers (ERL 100: ERS 100) and crystalline Tf and G. Reduced crystalline advocates increased mechanical behavior of fabricated scaffolds which would be an enviable stipulation for design biomedical gauge and bandage.

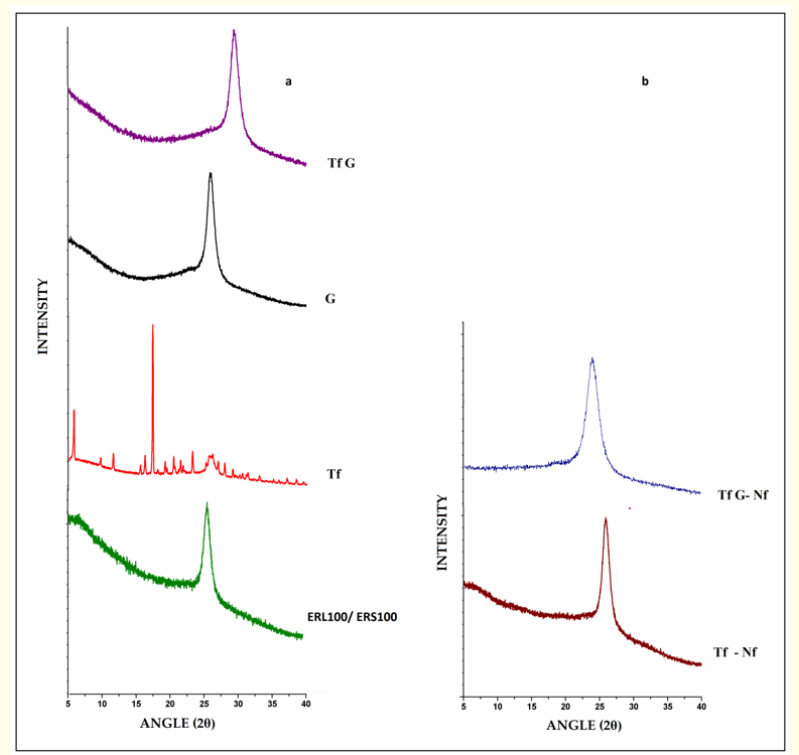

Figure 3: Compiled XRD portrait of drug Tf, graphene nanoplatelets G, Tf-G nanoconjugate (a) and fabricated scaffolds (b).

DSC curve of a blend of polymers ERL 100/ERS 100 showed glass transition temperature at $64.86^{\circ} \mathrm{C}$. Endothermic peaks at $161.94^{\circ} \mathrm{C}$ and $271.92^{\circ} \mathrm{C}$ were visible due to change in heat capacity and shallow peak at $343.27{ }^{\circ} \mathrm{C}$ exhibiting slow transformation along with less enthalpy change. Drug Tf showed different peaks of $113.23^{\circ} \mathrm{C}, 162.99^{\circ} \mathrm{C}, 251.72^{\circ} \mathrm{C}, 303.28^{\circ} \mathrm{C}$ and $344.72^{\circ} \mathrm{C}$, showing polymorphism. The sharp peak of $\mathrm{Tf}$ at $113.23^{\circ} \mathrm{C}$ revealed its melting point and fast transformation with larger enthalpy change
(Figure 4a). Developed nanoconjugate Tf G showed better thermal stability as compared to drug Tf, attributed to the formation of a conjugate with high thermal stable G. Moreover, in figure 4b, DSC of a more thermostable TfG-Nf was seen exhibiting distinct endothermic peaks at $210^{\circ} \mathrm{C}$ and $500^{\circ} \mathrm{C}$ respectively.
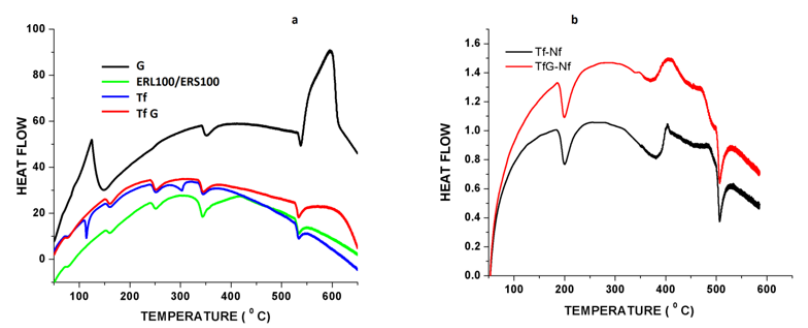

Figure 4: Differential scanning calorimeter (DSC) of individual ingredients (a) and scaffolds (b).

\section{Surface morphology}

Morphology of fabricated scaffolds exposed successfully electrospinnability of ERL100/ERS100 dispersion without apparent particles and beads over scaffolds (Figure 5). Herein, essential electrospinning factors such as controlled flow rate, optimum voltage, and the adequate distance between the syringe and collector were kept constant, therefore significant differences in the surface morphology of scaffolds are related to the intrinsic properties of polymeric dispersion loaded with Tf, G, and Tf G. Framework size analysis was performed through Image plus 6 software and almost symmetric bell-shaped graphs were obtained which emulated uniformity of creation. Their reduced fiber diameter reflected enhanced surface area and inherent small pores. The average fiber dimension of scaffolds Nf, Tf- Nf, G-Nf, and TfG-Nf were recorded as $76.5 \pm 15 \mathrm{~nm}, 302.6 \pm 50 \mathrm{~nm}, 213 \pm 85 \mathrm{~nm}$ and $105.6 \pm 15 \mathrm{~nm}$ respectively (Figure 5). Nf showed the smallest average diameter owing to a homogenous polymeric blend in selected solvents and uniformly electrospinning. Fabiola 2016 discussed narrower the diameter of fibers profound the surface region and therefore improved strength and stiffness. An amplified diameter of Tf- Nf might be due to either high drug entrapment or inadequate evaporation of the solvent (methanol and dimethylacetamide) from collector of electro-spinning machine. FE-SEM image of G-Nf showed 
elongated, regular and defect free scaffolds which focused new arena to researchers for innovative surgical dressing materials to cure microbial wound.

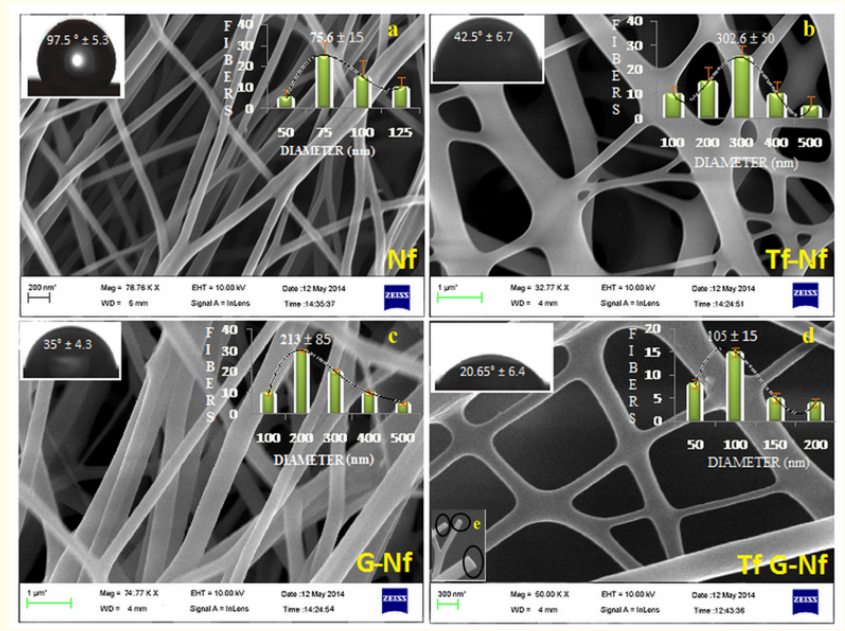

Figure 5: FESEM images and fiber size analysis of electrospunned scaffolds (Nf, Tf Nf, G Nf, and TfG- Nf).

By contrast topography of TfG-Nf echoed semitransparent, branched/mesh-like assembly that would be an ideal architecture for scaffolds designed for management of Tinea infections as it simulated extracellular matrix (ECM) which would support skin regeneration. Presence of voids/pockets in their surface supposed to provide sufficient gaseous exchange for cells with their environment and absorb sufficient exudates to maintain cellular functionalities, leading to better patient compliance. Addition of PEG 400 into the polymeric dispersion, obviously imparted uniformity and flexibility to the scaffold, must be seen in FESEM image of TfG-Nf.

Huang et al. 2012 described the consequence of ambient processing temperature and apparent polymeric viscosity on the shape (flat/circular) of a cross-section of nanofibers. Figure $5 \mathrm{e}$ showed nonporous, circular edges of cross-sectional Tf G-Nf scaffolds at $37^{\circ} \mathrm{C}$ ambient temperature and 22 cps polymeric viscosity, which might be accountable for steady diffusion of media through the core of its surface required for controlled release of therapeutic agent [42].
Additionally, the inset of FESEM images of all scaffolds predicted average WCA estimated through sessile drop method. Polymethacrylates eudragit polymers contained inherent water permeable property which was also supposed to be hiked with hydrophilic PEG 400 thus favored more wettability of scaffolds. The least contact angle $\left(20.65^{\circ} \pm 6.4\right)$ of TfG-Nf specified its kinship with produced exudation during contagion. Though presence of abundant pockets/mesh on the surface of scaffolds would enable adequate dispersal of exudate and would facilitate desired adhesion over the infected skin and persuade tissue proliferation at a higher degree leading to upsurge cure rate.

Swelling behavior of designed scaffolds was examined by the gravimetric method in $\mathrm{pH} 6.8$ buffer media. The degree of swelling would influence the drug release behavior of these scaffolds. Swelling behavior of PEG incorporated Eudragit polymers (ERL100/ ERS100) would allow a mechanical entanglement between the scaffolds and the infected surface by revealing its bioadhesive sites for electrostatic interaction or hydrogen bonding. This is possibly due to the customized water permeability of polymers, and cosolvency of PEG 400 which allowed each chain to hydrate freely, owing to superb swelling capability. Figure 6a compared outcomes of swelling capability of fabricated scaffolds up to $24 \mathrm{hrs}$, TfG-Nf showed better swelling phenomenon owing to the presence of plentiful pockets, which might be occupied sufficient medium (Figure $6 \mathrm{~b}$ ). However, consequences of swelling index were found to be declined after $8 \mathrm{hrs}$, the reason behind this could be turndown integrity of scaffolds in the experimental media.

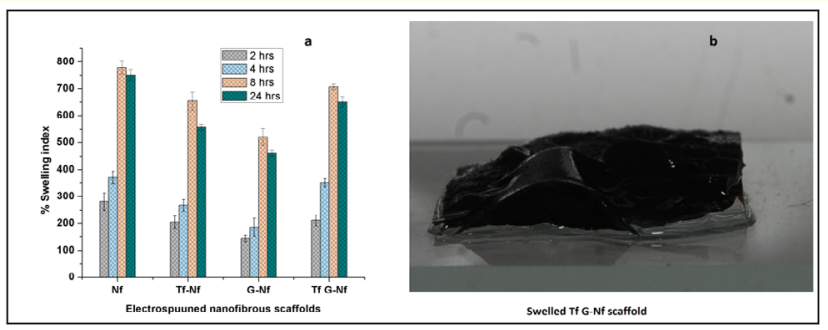

Figure 6: Comparative swelling index of fabricated Nf, Tf Nf, G Nf, and TfG- Nf scaffolds (a) Swelled TfG-Nf (b).

\section{In vitro drug release}

Preceding investigation on tinea infection demonstrated that the pathogen changes skin $\mathrm{pH}$ from acidic to alkaline $\mathrm{pH}$, therefore 
in vitro study was performed in both media (acidic and alkaline) dissolution media to estimate drug release efficiency. Comparative percentage cumulative drug release (\% CDR) profile of drug laded scaffolds in variable release media (phosphate buffer $\mathrm{pH} 6.8$, acetate buffer $\mathrm{pH} 3.8$ and alkaline borate $\mathrm{pH} 9.0$ ) has been illustrated in figure 7. Higher drug release from TfG-Nf scaffolds suggested, primarily desorption of embedded therapeutic agent from their architecture that might be principal pathway as well as the ratelimiting step.

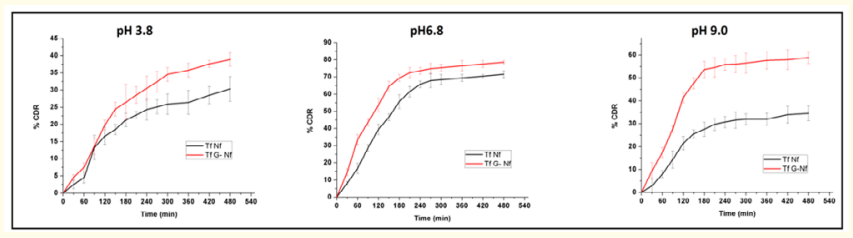

Figure 7: Comparative In vitro drug release from $\mathrm{Tf} \mathrm{Nf}$ and TfG- Nf scaffolds in the different release medium. Higher drug release from fabricated scaffolds TfG -Nf must be governed by their superior swelling capacity and sufficient permeation of dissolution media into their mesh structure that expedited drug diffusion from their surface.

TfG-Nf exhibited comparatively enhanced drug release within 2 hrs in the release media of phosphate buffer $\mathrm{pH} 6.8$, which was further found to be controlled up to $8 \mathrm{hrs}$. Initially, surged release of Tf was observed due to prompt release of embedded drug present on the surface of scaffolds that might be required for eliminating tinea at prelim phase. Furthermore, slothful drug release during 2-8 hrs favored consistent anti-tinea action of scaffolds. Incorporation of PEG 400 in polymeric solution not only expedites rapid hydration of scaffolds but also equal blend of ERL100/ERS100 would customize control drug release in provided dissolution medium. From figure 7 of comparative drug release outcomes summarized pattern of drug release from scaffolds TfG-Nf in order of phosphate buffer $\mathrm{pH}$ $6.8(78.9 \% \pm 3.4)>$ alkaline borate $\mathrm{pH} 9.0(58.89 \% \pm 2.6)>$ acetate buffer $\mathrm{pH} 3.8(38.89 \% \pm 2.3)$, concluding superior efficacy at the preliminary phase of tinea infections.

\section{Localized effect}

Localized effect of the electrospunned scaffolds was investigated against tinea infection causing T. rubrum and M. fulvum fun- gal strains. Figure 8 revealed that fabricated scaffolds eliminated colonies of fungal pathogens in SDA media through direct contact. The image (Figure 8) displayed the tendency of scaffolds to hold or entrap the bioactive compounds within their network. Such a nonleaching and localized characteristic led to an excellent stability for antitinea action, which also abolishes the distress of antifungal agents entering the surrounding environment (healthy tissue) to cause undesirable complications.
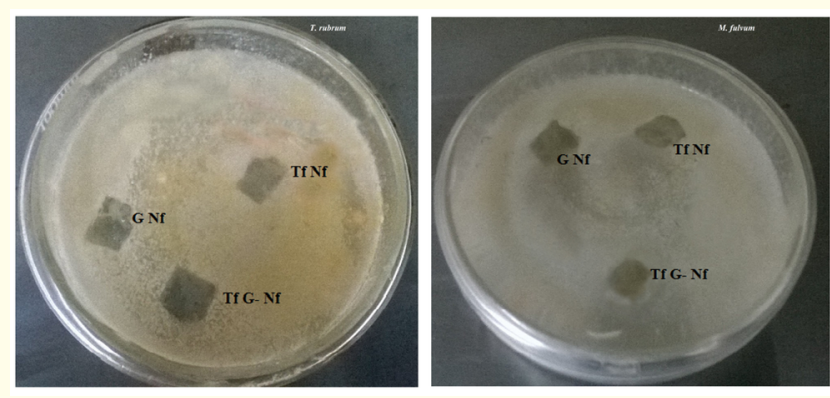

Figure 8: Localized zone exhibited by fabricated scaffolds on SDA culture plates of T. rubrum and M. fulvum.

\section{In vitro antifungal assay}

In vitro antifungal efficacy of fabricated scaffolds was evaluated quantitatively through broth dilution technique. Experimental observation for minimum inhibitory concentrations (IC 50 and MIC 90) of scaffolds against selected tinea pathogens was compiled in table 1. Amid scaffolds, TfG-Nf showed highest vulnerability towards strain of T. rubrum i.e. least MIC for T. rubrum. Outcomes exposed antidermatophyte activity of G-Nf also, against selected dermatophytes which shed light for exploration of graphene for designing dressing materials to mitigate topical skin bugs.

Graphs sketched between percentage growth inhibitions versus time wrapped up that electrospunned scaffolds possessed antitinea activity towards test pathogens till $96 \mathrm{hrs}$. In figure 9, Tf- Nf exhibited $95.981 \%$ and $89.789 \%$ growth inhibition against $M$. fulvum and T. rubrum whereas TfG-Nf showed enhanced $99.356 \%$ and 95.421\% growth inhibition against above-cited dermatophytes for $72 \mathrm{hrs}$ after that continuous anti- tinea action was revealed for 96 hrs. The outcomes supported dominant anti-tinea action towards pathogen T. rubrum, the causative agent of athletes foot. The ra- 


\begin{tabular}{|c|c|c|c|}
\hline $\begin{array}{c}\text { Tinea } \\
\text { pathogens }\end{array}$ & $\begin{array}{c}\text { Fabricated } \\
\text { scaffolds }\end{array}$ & $\begin{array}{l}\mathrm{MIC} * 50 \\
(\mathrm{mg} / \mathrm{ml})\end{array}$ & $\begin{array}{c}\text { MIC } \$ 90(\mathrm{mg} / \\
\mathrm{ml})\end{array}$ \\
\hline \multirow{3}{*}{ T. rubrum } & Tf-Nf & 0.092 & 0.851 \\
\hline & G-Nf & 0.112 & 0.792 \\
\hline & TfG-Nf & 0.045 & 0.257 \\
\hline \multirow{3}{*}{ M. fulvum } & Tf-Nf & 0.229 & 1.572 \\
\hline & G-Nf & 0.476 & 1.888 \\
\hline & TfG-Nf & 0.135 & 0.978 \\
\hline \multicolumn{4}{|c|}{$\begin{array}{l}\text { *Mean inhibitory concentration (MIC) } 50 \% \text { growth } \\
\text { inhibition of dermatophytes }\end{array}$} \\
\hline
\end{tabular}

Table 1: MIC estimated through microdilution method against dermatophytes (T. rubrum and M. fulvum) after $96 \mathrm{hrs}$.

tionale behind consequences must be attributed to mingle action of both drug Tf and G. Gathered results also opened scope for suppression of other 'Tinea' bugs befell to living beings. Although, antifungal activity against T. rubrum exhibited a lag phase for growth inhibition which was found to be increase with time (Figure 9a), the reason behind this might be its resistant nature for applied test samples. Findings also revealed that scaffolds G-Nf possessed antifungal activity, which explores its potential application in superficial infections. The present research envisaged this latent action of graphene might be by the virtue of its sharp edges and substantial cell killing properties [43].

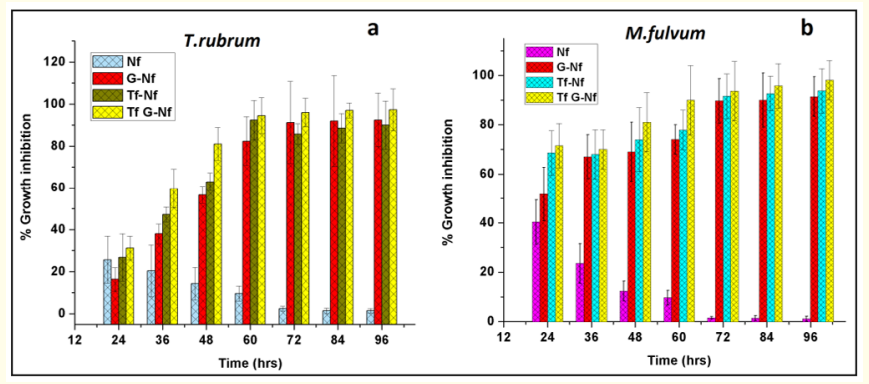

Figure 9: In vitro percentage growth inhibition displayed by fabricated scaffolds against tinea pathogens analyzed by SoftMax Pro software (SpectraMax Plus384).
Further, correlation coefficient $\left(\mathrm{r}^{2}\right)$ was computed by applying linear equation to investigate inhibition/susceptibility of all electrospunned scaffolds towards virulent strain T. rubrum and M. fulvum (Figure 10). Obtained data emphasized pronounced activity of TfG-Nf against T. rubrum having maximum linear regression $\left(\mathrm{r}^{2}=\right.$ 0.973) and suggested potential application of nano dimensional scaffolds for the treatment of Tinea infections.

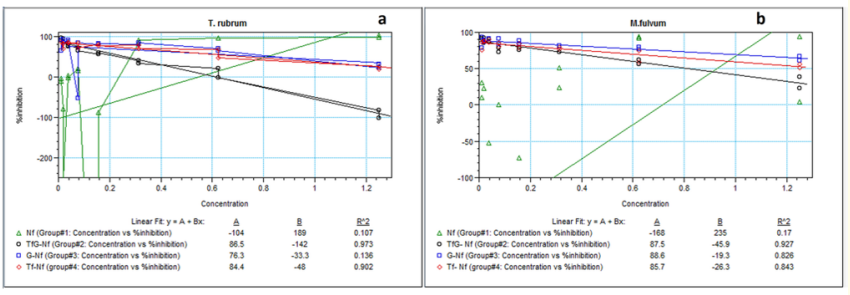

Figure 10: Linear graph plotted between concentration and percentage inhibition against pathogens (T. rubrum and $M$. fulvum). Records produced by SoftMax Pro software, model SpectraMax Plus384.

\section{In vivo studies}

After inoculation of pathogen T. rubrum on Swiss albino mice, initially small scaly patches or erythematous symptoms appeared on the dorsal surface of mice that were constantly amplified on successive days. The average lesion score was ranged from 2-3 as each infected group was severely erythematous after five days of inoculation. Application of fabricated scaffolds on infected surface was carried out for seven days and measurement of infection could be carried out by scoring them. The treatment scores were given to each mice group as 0 uncured), 1 (1/4 cured), 2 ( $1 / 2$ cured), 3 (2/3 cured) and 4 (all cured).

Figure 11 compiled images of in vivo antifungal study for seven days which estimated the treatment score for Group I (control) was zero as fungal infection progressively spread over the dorsal surface (figure $11 \mathrm{a}-\mathrm{d}$ ). Group II (treated with marketed formulation) cured the fungal infection but there were signs of crusty appearance (figure 11h) on the infected site hence scored 3. Group III (treated with the blank scaffolds, Nf) scored 1 as the infection was not cured significantly (figure 11, l) whereas Group IV (Tf loaded scaffolds, Tf- Nf) and group V (G loaded scaffolds, G-Nf) scored 2 
( $50 \%$ cure) as there were indication of prominent crusty scales on infected site. Group VI (TfG loaded scaffolds, TfG-Nf) scored 4 or $100 \%$ cure, as complete recovery from infection, faded all lesions (figure $11 \mathrm{x}$ ) with signs of appearance of new hair. T. rubrum would be killed or inhibited with the initial release of bioactive antifungal from scaffold, further its sustained release could help to prevent supplementary infection. Enhanced in vivo efficiency of TfG-Nf might be attributed due to continuous and controlled diffusion of Tf G conjugate from Tf G-Nf scaffolds that worked as a depot at the infected site.

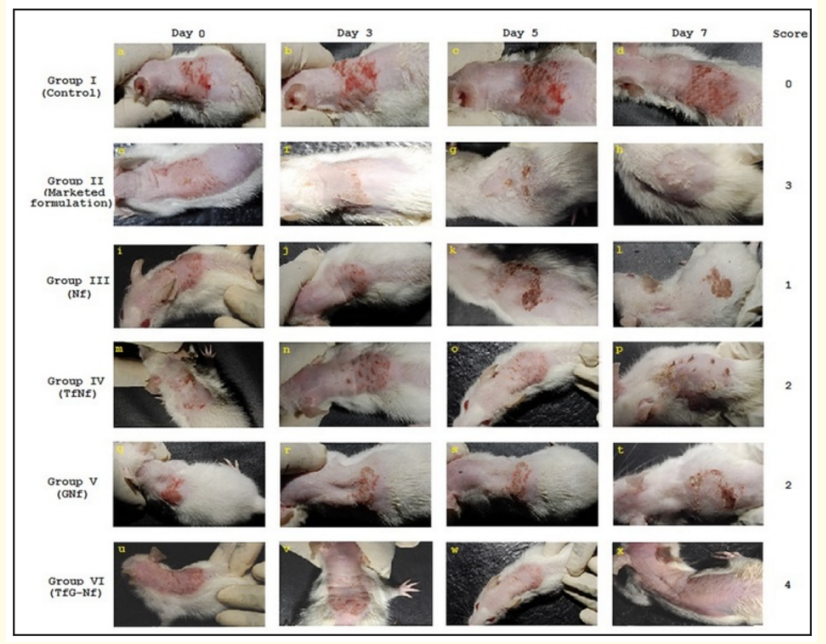

Figure 11: Comparative in vivo study on each infected group and their treatment score.

The in vivo study confirmed that graphene and its developed conjugate (Tf G) can treat T. rubrum induced topical infection on albino mice and also correlated well with findings of in vitro drug release and antifungal broth microdilution assay. Hence proved to have property to check the fungal growth, cure the topical infection, and could also represent a possibly better alternative for the treatment of variable Tinea infections. At last to make sure complete eradication of pathogen (T. rubrum) from the infected site of mice, a minor portion of skin was collected, cultured on SDA plate and allowed for incubation (7 days) to spot any kind of dermatophyte colony.

\section{Conclusion}

PEG assimilated Eudragit polymeric scaffolds were auspiciously fabricated and loaded with TfG conjugate to amplify antifungal efficacy towards disastrous Tinea infections. The inclusion of PEG 400 in polymeric mixture efficiently prevented particle-particle agglomeration and produced stable polymeric dispersion to render ease of electrospinning; resultant defect free scaffolds with sufficient flexibility could be fabricated. Electrospunned TfG-Nf mimicked ECM, found to be nonporous, semitransparent, and comprised pockets of nanoscaled dimension. The drug release profile of fabrications suggested a controlled rate diffusion mechanism. The nonwoven surface architect, excellent swelling index, expedient hydrophilicity, controlled drug release and remarked localized effect suggested promising application of fabricated TfG-Nf scaffolds for mitigation of diseases like Tinea pedis and Tinea corporis. In vivo study on Swiss albino mice exposed superb antifungal activity and escalated cure rate of TfG-Nf inoculated by contagious strain T. rubrum, the causative agent of Tinea pedis. The current study suggested the prospective application of nano dimensional $\mathrm{TfG}$-Nf for the management of wound healing and skin regeneration amid better patient compliance.

\section{Acknowledgments}

We thankfully acknowledge the authorities and staff members of SIDBI incubation center, IIT Kanpur and Botany Department and the University of Allahabad for generously granting all working facilities and extending their grudgeless cooperation. The authors are also thankful to Dr. Deepak Srivastava, Research Scientist, CDRI, Lucknow for his kind support to analyze samples.

\section{Bibliography}

1. Peres NT., et al. "Dermatophytes: host pathogen interaction and antifungal resistance". Anais Brasileiros de Dermatologia 85.5 (2010): 657-667.

2. Araujo CR., et al. "In Vitro susceptibility testing of dermatophytes isolated in Goiania, Brazil, against five antifungal agents by broth microdilution method". Revista do Instituto de Medicina Tropical de São Paulo 51.1 (2009): 9-12.

3. Bergus GR., et al. "Superficial tinea infections". American Family Physician 48 (1993): 259-268. 
4. Lakshmipathy DK., et al. "Review on dermatomycosis: pathogenesis and treatment”. Natural Science 2.7 (2010): 726-731.

5. Kaplan W., et al. "Ringworm in the dog caused by Trichophyton rubrum”. Veterinary Medicine 53 (1958): 139-142.

6. Grappel SF., et al. "Immunology of dermatophytes and dermatophytosis”. Bacteriology Reviews 38.3 (1974): 222-250.

7. Perea S., et al. "Antifungal resistance in pathogenic fungi". Clinical Infectious Diseases 35.9 (2002): 1073-1080.

8. Berdy J., et al. "Bioactive Metabolites from Microorganisms". Elsevier, Amsterdam (2005): 3-25.

9. Dhivya S., et al. "Wound dressings- a review". Biomedicine 5.4 (2015): 22-29.

10. Camposeo A., et al. "Local mechanical properties of electrospun fibres correlate to their internal nanostructure". Nano Letter 13.11 (2013): 5056-5062.

11. Lin J., et al. "Co-electrospun nanofibrous membranes of collagen and zein for wound healing". ACS Applied Materials and Interfaces 4.2 (2012): 1050-1057.

12. Iwata K., et al. "In vitro thiocarbamate resistance of trichophyton mentagrophytes". Chemotherapy 38.5 (1990): 435-443.

13. Yamaguchi H., et al. "Therapeutic efficacy of a topical tolnaftate preparation in guinea pig model of tinea pedis". Japanese Journal of Antibiotics 54.6 (2001): 323-330.

14. Akhavan 0., et al. "Toxicity of graphene and graphene oxide nanowalls against bacteria". ACS Nano 4.10 (2010): 57315736.

15. Kalbacova M., et al. "Graphene substrates promote adherence of human osteoblasts and mesenchymal stromal cells". Carbon 48.15 (2010): 4323-4329.

16. Nayak TRS., et al. "Graphene for controlled and accelerated osteogenic differentiation of human mesenchymal stem cells". ACS Nano 5.6 (2011): 4670-4678.

17. Ryoo., et al. "Behaviours of NIH-3T3 fibro-blasts on graphene /caron nanotubes: proliferation, focal adhesion, and gene transfection studies". ACS Nano 4.11 (2010): 6587-6598.
18. Novoselov KS., et al. "Electric field effect in atomically thin carbon films". Science 306.5696 (2004): 666-669.

19. Bolotin KI., et al. "Ultrahigh electro mobility in suspended graphene". Solid State Communication 14 (2008)6: 351-355.

20. Sundaray B., et al. "Preparation and characterization of electrospun fibres of poly (methyl methacrylate) single walled carbon nanotube nanocomposites". Journal of Engineered Fibers and Fabrics 3.4 (2008): 39-45.

21. Kam NWS., et al. "Nanotube molecular transporters internalization of carbon nanotube- protein conjugates into mammalian cells". Journal of the American Chemical Society 126.22 (2004): 6850-6851.

22. Li N., et al. "Flexible graphene- based lithium ion batteries with ultrafast charge and discharge rates". Proceedings of the $\mathrm{Na}$ tional Academy of Sciences of the United States of America 109 (2012): 17360-17365.

23. Pandey H. "Controlled drug release characteristics and enhanced antibacterial effect of graphene nanosheets containing gentamicin sulfate". Nanoscale 3 (2011): 4104-1408.

24. Kumari P., et al. "Formulation and evaluation of tolnaftate microsponges loaded gels for treatment of dermatophytosis". European Journal of Pharmaceutical and Medical Research 4.6 (2017): 326-335

25. Dillen K., et al. "Evaluation of Ciprofloxacin Loaded Eudragit RS 100 or RL 100/plga Nanoparticles". International Journal of Pharmaceutics 314 (2006): 72-82.

26. Jiang X., et al. "Plasmid templated shape control of condensed DNA- block copolymer nanoparticles". Advances in Materials 25 (2013): 227-232.

27. Vardharajula S., et al. "Functionalized carbon nanotubes: biomedical applications". International Journal of Nanomedicine 7 (2012): 5361-5374.

28. Liu Z., et al. "Pegylated nano- graphene oxide for delivery of water insoluble cancer drugs". Journal of the American Chemical Society 130.33 (2008): 10876-10877. 
29. Ravelli D., et al. "PEGylated carbon nanotubes: preparation, properties and applications". RSC Advances 3 (2013): 1356913582.

30. Misra SK., et al. "Carbon Allotrope Graphene: Superstar In Nano- World". International Journal of Advances in Pharmaceutical Sciences 2.1 (2013): 1-4.

31. Karthikeyana K., et al. "Electrospun zein/eudragit nanofibres based dual drug delivery system for the simultaneous delivery of aceclofenac and pantoprazole". International Journal of Pharmaceutics 438 (2012): 117-122.

32. Lin J., et al. "Co-electrospun nanofibrous membranes of collagen and zein for wound healing". ACS Applied Materials and Interfaces 4 (2012): 1050-1057.

33. Huang FL., et al. "Dynamic wettability and contact angles of poly (vinylidene fluoride) nanofiber membranes grafted with acrylic acid". eXPRESS Polymer Letters 4.9 (2010): 551-558.

34. Castelli F., et al. "Flurbiprofen release from Eudragit RS and RL aqueous nanosuspensions: a kinetic study by DSC and dialysis experiments". AAPS Pharm Sci Tech 3.2 (2002): 1-8.

35. Martinez -Rossi NM., et al. "Role of $\mathrm{pH}$ in the pathogenesis of dermatophytoses". Mycoses 55 (2012): 381-387.

36. Ahmed A., et al. "Nanofiber composites containing N-heterocyclic carbene complexes with antimicrobial activity". International Journal of Nanomedicine 7 (2012): 2829-2832.

37. Pandey S., et al. "Synthesis and characterization of grapheneusnic acid conjugate microspheres and its antibacterial activity against Staphylococcus aureus". IJPSR 10.2 (2019): 10001008.

38. Chen J., et al. "Graphene oxide exhibits broad-spectrum antimicrobial activity against bacterial phytopathogens and fungal conidia by interwining and membrane perturbation". Nanoscale 6.3 (2014): 1879-1889.

39. Misra SK., et al. "Tolnaftate loaded polyacrylate electrospun nanofibers for impressive regimen on dermatophytosis". Fibers 5 (2017): 41-52.
40. Sharma N., et al. "Smart Graphene-Keto Nano hybrids Loaded Therapeutics for Effective Management Dermatophytosis". Drug Delivery Letter 9.1 (2019): 21-28.

41. Padhan DK., et al. "In Vivo antifungal activity of Accmella essential oil on a dermatomycotic strain Trichophyton mentagrophytes (MTCC-7687)". Pelagia Research Library, Der Pharmacia Sinica 5.1 (2014): 40-44.

42. Huang C., et al. "Fiber, shape structure and surface texture". Journal of Nanomedicine (2012): 1-7.

43. Misra SK., et al. "Tolnaftate- graphene composite loaded nanoengineered electrospun scaffods as efficient therapeutic dressing material for regimen of dermatomycosis". Applied Nanoscience 8.7 (2018): 1629-1640.

Volume 5 Issue 7 July 2021

(C) All rights are reserved by Shashi Kiran Misra., et al. 